\title{
CORRIGENDUM
}

\section{Propagation characteristics of Hermite-cosh-Gaussian laser beam in a rippled density plasmas-CORRIGENDUM}

\section{S. KAUR, M. KAUR, R. KAUR AND T. S. GILL}

doi:10.1017/S026303461600080, Published by Cambridge University Press, 4 January 2017.

The version of this manuscript originally published contained a error in Eq. (12b).

$$
\begin{aligned}
& {\left[1+\frac{\xi \alpha_{2} q^{\prime} \sin \left(q^{\prime} \xi\right)\left[\omega_{\mathrm{p} 0}^{2} / \gamma \omega^{2}\right]}{2\left(1-\left(\omega_{\mathrm{p} 0}^{2} / \gamma \omega^{2}\right)-\left(\omega_{\mathrm{p} 0}^{2} / \gamma \omega^{2}\right) \alpha_{2} \cos \left(q^{\prime} \xi\right)\right)}\right]\left(\frac{\alpha_{2} q^{\prime} \sin \left(q^{\prime} \xi\right)\left[\omega_{\mathrm{p} 0}^{2} / \gamma \omega^{2}\right](\partial f / \partial \xi)}{2\left(1-\left(\omega_{\mathrm{p} 0}^{2} / \gamma \omega^{2}\right)-\left(\omega_{\mathrm{p} 0}^{2} / \gamma \omega^{2}\right) \alpha_{2} \cos \left(q^{\prime} \xi\right)\right)}\right)} \\
& -\frac{\xi \alpha_{2} q^{\prime} \sin \left(q^{\prime} \xi\right)\left[\omega_{\mathrm{p} 0}^{2} / \gamma \omega^{2}\right]}{2\left(1-\left[\omega_{\mathrm{p} 0}^{2} / \gamma \omega^{2}\right]-\left[\omega_{\mathrm{p} 0}^{2} / \gamma \omega^{2}\right] \alpha_{2} \cos \left(q^{\prime} \xi\right)\right)} \frac{1}{f}\left(\frac{d f}{d \xi}\right)^{2}-\frac{\left(4-4 b^{2}\right)}{f^{3}} \\
& -\frac{8 \alpha E_{0}^{2}}{f^{3}}\left(\frac{\omega_{\mathrm{p} 0}^{2}}{\omega^{2}}+\frac{\omega_{\mathrm{p} 0}^{2}}{\omega^{2}} \alpha_{2} \cos \left(q^{\prime} \xi\right)\right)\left(\frac{\omega r_{0}}{c}\right)^{2}\left(2-b^{2}\right) e^{b^{2} / 2}=0 .
\end{aligned}
$$

should be replaced by:

$$
\begin{aligned}
& {\left[1+\frac{\xi \alpha_{2} q^{\prime} \sin \left(q^{\prime} \xi\right)\left[\omega_{p 0}^{2} / \gamma \omega^{2}\right]}{2\left(1-\left[\omega_{p 0}^{2} / \gamma \omega^{2}\right]-\left[\omega_{p 0}^{2} / \gamma \omega^{2}\right] \alpha_{2} \cos \left(\mathrm{q}^{\prime} \xi\right)\right)}\right] \frac{d^{2} f}{d \xi^{2}}+\left[1+\frac{\xi \alpha_{2} q^{\prime} \sin \left(q^{\prime} \xi\right)\left[\omega_{p 0}^{2} / \gamma \omega^{2}\right]}{2\left(1-\left[\omega_{p 0}^{2} / \gamma \omega^{2}\right]-\left[\omega_{p 0}^{2} / \gamma \omega^{2}\right] \alpha_{2} \cos \left(\mathrm{q}^{\prime} \xi\right)\right)}\right]} \\
& \left(\frac{\alpha_{2} q^{\prime} \sin \left(q^{\prime} \xi\right)\left[\omega_{p 0}^{2} / \gamma \omega^{2}\right](\partial f / \partial \xi)}{2\left(1-\left[\omega_{p 0}^{2} / \gamma \omega^{2}\right]-\left[\omega_{p 0}^{2} / \gamma \omega^{2}\right] \alpha_{2} \cos \left(\mathrm{q}^{\prime} \xi\right)\right)}\right)-\left(\frac{\xi \alpha_{2} q^{\prime} \sin \left(q^{\prime} \xi\right)\left[\omega_{p 0}^{2} / \gamma \omega^{2}\right]}{2\left(1-\left[\omega_{p 0}^{2} / \gamma \omega^{2}\right]-\left[\omega_{p 0}^{2} / \gamma \omega^{2}\right] \alpha_{2} \cos \left(\mathrm{q}^{\prime} \xi\right)\right)}\right) \frac{1}{f}\left(\frac{d f}{d \xi}\right)^{2} \\
& -\frac{\left(4-4 b^{2}\right)}{f^{3}}-\frac{8 \alpha E_{0}^{2}}{f^{3}}\left(\frac{\omega_{p 0}^{2}}{\omega^{2}}+\frac{\omega_{p 0}^{2}}{\omega^{2}} \alpha_{2} \cos \left(q^{\prime} \xi\right)\right)\left(\frac{\omega r_{0}}{c}\right)^{2}\left(2-b^{2}\right) e^{b^{2} / 2}=0
\end{aligned}
$$

\section{REFERENCE}

KaUR, S., KaUR, M., KaUR, R. \& GILL, T.S. (2017). Propagation characteristics of Hermite-cosh-Gaussian laser beam in a rippled density plasmas. Laser Part. Beams 35, 100-107. 